\title{
CUCKOO SEARCH ALGORITHM FOR OPTIMAL PLACEMENT AND SIZING OF STATIC VAR COMPENSATOR IN LARGE-SCALE POWER SYSTEMS
}

\author{
Khai Phuc Nguyen ${ }^{1}$, Goro Fujita ${ }^{1}$, Vo Ngoc Dieu ${ }^{2}$ \\ ${ }^{1}$ Power System Laboratory, \\ Shibaura Institute of Technology, Tokyo, Japan \\ ${ }^{2}$ Division of Power system Engineering, \\ Ho Chi Minh University of Technology, Ho Chi Minh City, Vietnam
}

\begin{abstract}
This paper presents an application of Cuckoo search algorithm to determine optimal location and sizing of Static VAR Compensator. Cuckoo search algorithm is a modern heuristic technique basing Cuckoo species' parasitic strategy. The Lévy flight has been employed to generate random Cuckoo eggs. Moreover, the objective function is a multiobjective problem, which minimizes loss power, voltage deviation and investment cost of Static VAR Compensator while satisfying other operating constraints in power system. Cuckoo search algorithm is evaluated on three case studies and compared with the Teaching-learning-based optimization, Particle Swarm optimization and Improved Harmony search algorithm. The results show that Cuckoo search algorithm is better than other optimization techniques and its performance is also better.
\end{abstract}

Keywords: Cuckoo search algorithm, optimal placement and sizing, shunt VAR compensator, optimal power flow, FACTS

\section{Introduction}

In reconfiguration of the electric power system, Flexible AC transmission system (FACTS) devices play an important role. FACTS give many benefits of dynamic stability and steady-state controls of a power system. Among FACTS devices, Static VAR Compensator (SVC) is widely used because of its low cost, easy control and good performance. The first required problem to install SVC or other FACTS devices in power system is to determine place and size of them.

In literature, this problem has been mentioned in various ways. For example, Y. Del Valle et al. applied the particle swarm optimization for finding size and location of a Static Compensator (STAT-
$\mathrm{COM}$ ) to improve the voltage profile of Brazilian power system [1]. In Taiwan, Huang C.H. et al. employed four various FACTS devices to save active power of generators and enhance voltage profile. The optimal solution given by Harmony Search algorithm is better than methods [2]. Another research of Pisica et al. proposed a multi-objective function to determine the optimal placement and size of a SVC device [3]. The multi-objective function includes the power loss, the voltage deviation and the investment cost of SVC. They solved this problem by a version of genetic algorithm. Following this approach, Reza Sirjani et al. proposed an improved version of the Harmony search algorithm to solve the problem $[4,5]$. On summary, all of above studies successfully use evolutionary meth- 
ods to determine optimal location and size of SVC or other FACTS devices.

However, each method can solve some problems effectively. Thus, the requirement to develop a new optimization technique and apply it for various problems increasingly continues. Since 2009, Yang and Deb have been developing a modern natureinspired method, it names Cuckoo search algorithm [6, 7]. In 2013, a survey made by P. Civicioglu and $\mathrm{E}$. Besdok gives comparison of four methods: Cuckoo search, particle swarm optimization, differential evolution and artificial bee colony algorithms [8]. After obtaining 50 mathematical functions, they conducted that differential evolution and the Cuckoo search are quite better than particle swarm optimization and artificial bee colony algorithm. Furthermore, many researchers have applied this method for solving optimized problems in power system. For instance, Moravej, Z., \& Akhlaghi, A. basing on Cuckoo search give optimal location of distributed generators in distribution network [9]. Vo D.N. et al. proposed optimal commitment of thermal generators in power system [11]. Ahmed, J., \& Salam, Z. applied Cuckoo search for maximum power tracking for photovoltaic modules [10].

In this paper, we propose Cuckoo search algorithm to solve the multi-objective function for optimal SVC devices in electrical power system. It also gives a comparison between Cuckoo search algorithm and other methods. Three systems of IEEE tested cases are obtained to figure out the effect of the proposed method when increasing search space. The first benchmark is the modified IEEE 30-bus system with five candidate SVC devices. The second case study is the IEEE 57-bus system with six candidate SVC devices. The last case study is the IEEE 118-bus system considering 10 candidate SVC devices.

This paper includes six parts. Current part provides a literature review about applications of SVC in the electric power system and Cuckoo search algorithm. The second part describes three objectives and regular operational constraints of this problem. The next part shows original pseudo codes of Cuckoo search algorithm. In the forth part, we describes our implementation of Cuckoo search algorithm for this problem. Numerical results are shown in the fifth part and the last part is our conclusion and future work.

\section{Objectives and operational con- straints}

\subsection{Objectives}

The problem of optimal placement and sizing of SVC is described as a multi-objective problem. This problem is to minimize power losses, voltage deviations and investment cost. Where the objectives of decreasing power losses and voltage deviations are technical objectives, while the investment cost is an economic one.

\subsubsection{The active power losses}

The total power loss in a power system is given in literature as:

$$
\begin{aligned}
& P_{\text {loss }}=\sum_{l=1}^{b r} R_{l} I_{l}^{2}=\sum_{i=1}^{b} \sum_{\substack{j=1 \\
i \neq j}}^{b}\left[V_{i}^{2}+V_{j}^{2}-\right. \\
& -2 V_{i} V_{j} \cos \left(\delta_{i}-\delta_{j}\right) Y_{i i} \cos \varphi_{i j}
\end{aligned}
$$

where $b r$ and $b$ are the number of lines and buses, respectively; $R_{l}$ is the resistance of line $l_{t h} ; I_{l}$ is the current through line $l^{\text {th }} ; V_{i}$ and $\delta_{i}$ are the magnitude and angle of voltage at the $i^{\text {th }}$ bus, respectively; $Y_{i j}$ and $\varphi_{i j}$ are the magnitude and angle of the line admittance between bus $i^{t h}$ and bus $j^{\text {th }}$, respectively.

\subsubsection{The voltage deviation}

The voltage deviation is a sum of voltage deviations at all buses in the power system from reference values. The below formula defines the voltage deviation objective:

$$
\Delta V_{\Sigma}=\sum_{i=1}^{b}\left(\frac{V_{r e f, i}-V_{i}}{V_{r e f, i}}\right)^{2}
$$

where $V_{\text {ref,i }}$ is the reference voltage at the $i^{\text {th }}$ bus.

\subsubsection{The investment cost}

The investment cost of each SVC device is a quadratic function of reactive power [12]. Thus, the total investment cost as below:

$$
C_{S V C}=\sum_{k=1}^{n} 0.0003 Q_{k}^{2}-0.3051 Q_{k}+127.38
$$

where $n$ is the number of installed SVC, $Q_{k}$ is injected reactive power of the $k^{\text {th }} \mathrm{SVC}$. 


\subsection{Operational constraints}

Optimizing placement and sizing of SVC needs to satisfy all of operational constraints such as the power balance constraint, limitation of bus voltages and limitation of transmission lines.

\subsubsection{Power balance constraint}

As other problems for operation in a power system, the balance of generating and demand powers must be satisfied at each node. Two below equations describe the balance of active and reactive powers in a power system:

$$
\begin{aligned}
& P_{G, i}-P_{D, i}=V_{i} \sum_{i=1}^{b}\left[V _ { j } \left[G_{i j} \cos \left(\delta_{i}-\delta_{j}\right)+\right.\right. \\
& +B_{i j} \sin \left(\delta_{i}-\delta_{j}\right) \\
& Q_{G, i}-Q_{D, i}=V_{i} \sum_{i=1}^{b}\left[V _ { j } \left[G_{i j} \sin \left(\delta_{i}-\delta_{j}\right)-\right.\right. \\
& -B_{i j} \sin \left(\delta_{i}-\delta_{j}\right)
\end{aligned}
$$

where $P_{G, i}$ and $Q_{G, i}$ are the active and reactive generating powers at the $i^{\text {th }}$ bus, respectively; $P_{D, i}$ and $Q_{D, i}$ are the active and reactive of demand powers at the $i^{\text {th }}$ bus, respectively. $G_{i j}$ and $B_{i j}$ represent the real and imaginary components of element $Y_{i j}$ of the admittance matrix, respectively.

\subsubsection{Limitation of SVC devices}

Each SVC device only works in a range of reactive power:

$$
Q_{i, \min } \leq Q_{i} \leq Q_{i, \max }
$$

\subsubsection{Limitation of bus voltages}

In order to keep the power system operate in stability and commit power quality, bus voltage at each bus must be maintained around a nominal value.

$$
V_{i, \text { min }} \leq V_{i} \leq V_{i, \text { max }}
$$

\section{Cuckoo search algorithm}

Basing on the parasitic reproduction strategy of Cuckoo species in nature, Yang and Deb developed a population-based optimization algorithm, named Cuckoo search algorithm. This method simulates the actions of the female Cuckoo bird to lay her egg into the neighbor's nest. This method also considers the probability that the host bird finds out and abandons the Cuckoo egg.

The process of Cuckoo search algorithm includes two separate stages. In the first stage, Cuckoo eggs are created and laid into the host bird's nest. Yang and Deb used the Lévy flight to create the Cuckoo eggs. The other stage is the probability of abandonment of Cuckoo eggs.

Using the Levy flight for Cuckoo search algorithm is the key distribution of Yang and Deb. The Lévy flight provides a random walk while the random step length is drawn from the Lévy distribution. The Lévy distribution is a continuous probability distribution for non-negative random variable. The formula of the Lévy distribution is below and Figure 1 shows the cumulative of the Lévy distribution with various values of $c$ and $\mu$ is zero.

$$
f(x ; \mu, c)=\sqrt{\frac{c}{2 \pi}} \frac{e^{-\frac{c}{2(x-\mu)}}}{(x-\mu)^{3 / 2}}
$$

where $\mu$ is the location parameter and $c$ is the scale parameter.

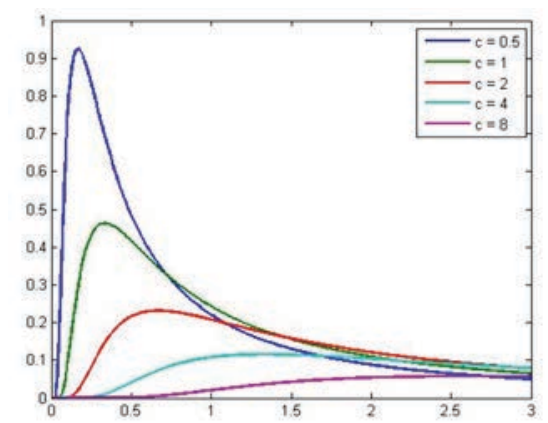

Figure 1. Cumulative of the Lévy distribution

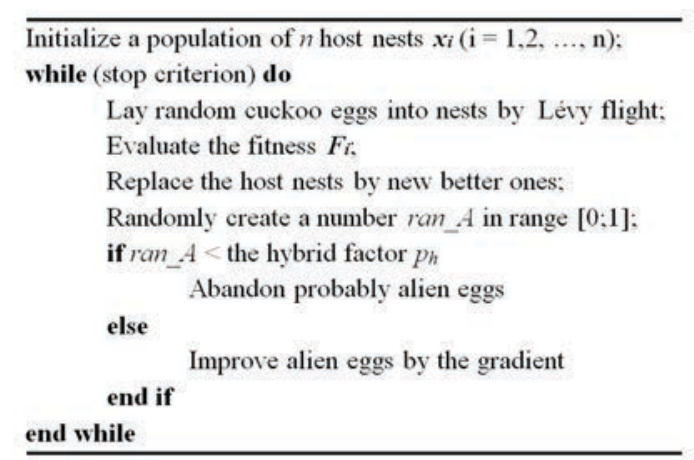

Figure 2. Pseudo code of Cuckoo search algorithm

However, it is too tricky to generate the step length for the Lévy flight. One of good strategies to generate the step length is the Mantegnas equations 
[13]. Following equations formulate the Mantegnas algorithm to generate the step length for Lévy flight and Figure 2 describes the pseudo code of Cuckoo search algorithm:

$$
\begin{gathered}
\text { step }=\frac{u}{v^{\frac{1}{\beta}}} ; \\
u=\operatorname{rand}() \cdot \sigma ; v=\operatorname{rand}() \\
\sigma=\left(\frac{\Gamma(1+\beta) \cdot \sin \left(\frac{\pi \cdot \beta}{2}\right)}{\Gamma\left(\frac{1+\beta}{2}\right) \cdot \beta \cdot 2^{\frac{\beta-1}{2}}}\right)^{\frac{1}{\beta}} ; \beta=\frac{3}{2}
\end{gathered}
$$

where $\Gamma()$ is the gamma function.

\section{Implementation and the fitness function}

\subsection{Solution vector}

A solution for this problem is a vector with $2 n$ elements; where $n$ is the number of candidate SVC devices. The first $n$ elements are positions of SVC devices. Each element is a natural number that represents the bus number where a SVC device is connected. The other elements are continuing values that represent optimal installed reactive power of SVC devices. Figure 3 shows the structure of a solution vector.

$$
\underbrace{\left\{x_{1}, x_{2} \ldots x_{n}, Q_{1}, Q_{2} \quad \ldots Q_{N}\right.}_{\text {Positions }}\}
$$

Figure 3. Structure of solution vector

With above structure of solution, it may lead the search engine to duplicated solutions. Table 1 shows an example of duplicated solutions. Two solutions actually give the same result that we need to install SVC at three buses $\{2,4$ and 7$\}$ with the same amount of injected reactive powers. Hence, to prevent this case, we proposed another constraint for positions of SVC as $x_{1}<x_{2}<\ldots<x_{n}$.
Table 1. Example of duplicated solutions

\begin{tabular}{|l|l|l|l|l|l|l|}
\cline { 2 - 7 } \multicolumn{1}{c|}{} & \multicolumn{3}{c|}{$\begin{array}{c}\text { Selected } \\
\text { buses }\end{array}$} & \multicolumn{3}{c|}{$\begin{array}{c}\text { Injected reactive } \\
\text { power (MW) }\end{array}$} \\
\hline Solution 1 & 2 & 4 & 7 & 44.95 & 40.69 & 23.76 \\
\hline Solution 2 & 4 & 7 & 2 & 40.69 & 23.76 & 44.95 \\
\hline
\end{tabular}

\subsection{Fitness function}

In order to describe three various objectives in a same mathematical function, we normalize each objective in a comparative manner with the base case (the system without SVC) and connect them together by weights. Equation (12) is the fitness function for this problem. With opinion that technical objectives are more important than economic one, the corresponding weights are set as $\alpha=0.4, \beta$ $=0.4, \eta=0.2$.

In order to handle operational constraints, we use penalty factors to combine with objective functions. The element balance_flag is a factor that equals to 0 if the power balance constraint is not violated and 1 otherwise. With the limits of bus voltages, we use a limited function, $V^{\lim }(x)$. Equation (13) describes the limited function. With the constraint for positions, we use a counter to find out the number of positions are violated. Through all tested cases, all penalty factors are 100 .

$$
\begin{aligned}
& F F=\alpha \frac{P_{\text {loss }}}{P_{\text {loss }, \text { base }}}+\beta \frac{\Delta V}{\Delta V_{\text {base }}}+\eta \frac{C_{S V C}}{C_{\max }}+ \\
& K_{p} \cdot \text { counter }+K_{p} \cdot \text { balance_flag }+ \\
& K_{p} \cdot \sum_{i=1}^{b}\left[V_{i}-V_{i}^{\lim }\left(V_{i}\right)\right]^{2} \\
& V^{\lim }(x)=\left\{\begin{array}{l}
x_{\max }, \text { if } x>x_{\max } \\
x, \text { if } x_{\min } \leq x \leq x_{\max } \\
x_{\min }, \text { if } x<x_{\min }
\end{array}\right.
\end{aligned}
$$

where:

- $P_{\text {loss }}:$ active power loss

$-\Delta V:$ voltage deviation index

$-C_{S V C}$ : total SVC cost

- $P_{\text {loss,base }}, \Delta V_{\text {base }}$ and $C_{\max }$ are the total base case active power loss in the network, the total base case voltage deviation and the maximum investment cost, respectively.

- $K_{p}$ : penalty factor 


\subsection{Limitation of solution vector and ini- tialization}

According to the structure of solution vector, the positions of candidate SVC devices cannot exceed the number of buses in the power system. Thus, $x_{\max }$ is the number of buses and $x_{\min }$ is equal to one. On the other hand, the injected reactive power of SVC devices cannot exceed its capacitor in the constraint (6). Similar to other populationbased methods, in the Cuckoo search algorithm, the nests also lay randomly between upper and lower bounds. However, for this problem, the first $n$ elements of nests are natural numbers. Hence, we use the round function round $(x)$ to return the value $x$ to the nearest natural number. Equation (14) and (15) describe the initialization of search space:

$$
\begin{array}{r}
N e s t_{i}=U p B+\operatorname{rand}() \cdot(U p B-L o w B) \\
N_{\text {est }}(1: n)=\operatorname{round}\left(\operatorname{Nest}_{i}(1: n)\right)
\end{array}
$$

where:

- Nest $t_{i}$ is the $i^{t h}$ nest in populations.

- $U p B$ and $L o w B$ are the upper and lower bound vectors, as following:

$$
\begin{aligned}
& U p B=\left\{x_{\max }, \ldots, x_{\max }, Q_{\max }, \ldots, Q_{\max }\right\} \\
& \text { LowB }=\left\{x_{\min }, \ldots, x_{\min }, Q_{\min }, \ldots, Q_{\min }\right\}
\end{aligned}
$$

\subsection{Generation of Cuckoo eggs via Lévy flight and discovery alien eggs}

As our above mention, the Cuckoo search algorithm includes two separate randomized processes. One of them is to generate Cuckoo eggs and another is to abandon alien eggs from the nests. In the first stage, from (9)- (11), a random step is created via Lévy flight. Following equations describe the process of laying Cuckoo eggs into nests.

$$
\begin{gathered}
n e w N e s t_{i}=\text { Nest }_{i}+\operatorname{rand}() . \Delta X_{i} \\
\Delta X_{i}=K_{1} . \text { step. }\left(\text { Nest }_{\text {best }}-\text { Nest }_{i}\right)
\end{gathered}
$$

where:

$-K_{1}:$ step-size factor

- step is the flying step generated by Lévy flight in (9).
- Nest best $_{\text {is }}$ the best solution.

In the discovering-alien-eggs stage, a new nest is generated randomly from populations. There is a probability rate $p_{a}$ to discover alien eggs. The new solutions can be found out as following way:

$$
\begin{array}{r}
\text { newNest }{ }_{i}=\text { Nest }_{i}+K . \Delta X_{i}^{\text {dis }} \\
K=\left\{\begin{array}{l}
1, \text { ifrand }()<p_{a} \\
0, \text { otherwise }
\end{array}\right. \\
\Delta X_{i}^{\text {dis }}=\operatorname{rand}()\left[\text { randperm }_{\left(\text {Nest }_{i}\right)-}\right. \\
-\operatorname{randperm}\left(\text { Nest }_{i}\right)
\end{array}
$$

where randperm $\left(\mathrm{Nest}_{i}\right)$ is the random perturbation for nests positions.

\subsection{Overall procedure}

The overall procedure for the implementation of the Cuckoo search algorithm to determine optimal placement and sizing of SVC devices:

- Step 1: Choose controlling parameters for the Cuckoo search algorithm, such as: the probability of discovering Cuckoo eggs, the number of nests $N P$ and the number of iterations $I t_{\max }$.

- Step 2: Create randomly initial nests currentNest.

- Step 3: Evaluate value of the fitness function $F F$ in (12), while using Newton-Raphson method for calculating the power flow.

- Step 4: Determine the best value of the fitness function FFbest and the best nest Nestbest. Set the iteration counter $k=1$.

- Step 5: Create Cuckoo eggs via Lévy flight, using (9)- (11), and new nests newNest as (18)(19).

- Step 6: Modify the eggs that violate the limitations of SVC device constraints and the limitation of bus numbers.

- Step 7: Evaluate the fitness function for new nests FFnew

- Step 8: Compare the new values FFnew to the current ones $F F$ to pick up the better nests. Update the currentNest, the best value of fitness function FFbest and the best nest Nestbest. 
- Step 9: Discovery Cuckoo eggs by random biased walks, create new nests newNest as (20)(22).

- Step 10: Modify the eggs that violate the limitations of SVC device constraints and the limitation of bus numbers.

- Step 11: Once again, evaluate the fitness function FFnew for new nests newNest

- Step 12: Update values of the fitness function $F F$ the currentNest, the best value of fitness function FFbest and the best nest Nestbest.

- Step 13: Check if the iteration counter $k$ is lower than the maximum iteration $I t_{\max }$, increase $k$ and return step 5. Otherwise, stop.

\section{Simulation results}

Cuckoo search algorithm has been applied to identify optimal placement and sizing of SVC devices in three various IEEE power systems. The first tested system is the modified IEEE 30-bus system. This system consists of six generators, 41 transmission lines and transformers. It supplies for 189.2 MW load power. Another larger system is also a standard IEEE system with 7 generators, 57 buses and 80 transmission lines-transformers. The last benchmark is the standard IEEE 118bus system. This system has 54 generators, 118 buses and 186 transmission lines-transformers. The obtained numerical results are compared with the Teaching-learning-based optimization (TLBO) [15, 16], self-organizing hierarchical particle swarm optimization with time-varying acceleration coefficients (SOHPSO-TVAC) [17] and Improved Harmony search algorithm (IHS) [18]. All applications are coded in Matlab 2015a and run in a personal computer with a $3 \mathrm{Ghz}$ Core 2 Duo processor and 4GB RAM. For each method, each benchmark is run 100 independent trials. In order to calculate power flow, we used the Newton-Raphson method by the Matpower toolbox [14]. Table 2 shows the dimension, size of population, number of iterations and selected parameters of Cuckoo search algorithm for each benchmark.
Table 2. Size of search space and number of iterations

\begin{tabular}{|l|c|c|c|}
\cline { 2 - 4 } \multicolumn{1}{l|}{} & $\begin{array}{c}\text { 30-bus } \\
\text { case }\end{array}$ & $\begin{array}{c}\text { 57-bus } \\
\text { case }\end{array}$ & $\begin{array}{c}\text { 118-bus } \\
\text { case }\end{array}$ \\
\hline $\begin{array}{l}\text { Number of can- } \\
\text { didate SVC }\end{array}$ & 5 & 6 & 10 \\
\hline $\begin{array}{l}\text { Number of pop- } \\
\text { ulation }\end{array}$ & 30 & 50 & 50 \\
\hline Iteration & 500 & 5000 & 1000 \\
\hline Probability $p_{a}$ & 0.8 & 0.7 & 0.9 \\
\hline
\end{tabular}

\subsection{Case study 1: IEEE 30-bus system}

Table 3. Numerical results of CSA and TLBO for IEEE 30-bus system

\begin{tabular}{|c|c|c|l|c|}
\cline { 2 - 5 } \multicolumn{1}{c|}{} & CSA & TLBO & $\begin{array}{l}\text { SOHPSO } \\
\text { TVAC }\end{array}$ & IHS \\
\hline Best & 1.4502 & 1.4502 & 1.4783 & 1.4626 \\
\hline Mean & 1.4630 & 1.4810 & 1.5217 & 1.4764 \\
\hline Worst & 1.4924 & 1.5089 & 1.5217 & 1.5139 \\
\hline SD & 0.0080 & 0.0139 & 0.0165 & 0.0160 \\
\hline
\end{tabular}

Table 4. Optimal solution of CSA in IEEE 30-bus case study

\begin{tabular}{|c|c|}
\hline Selected bus & Reactive power [MVar] \\
\hline 8 & 46.8054 \\
\hline 12 & 29.1442 \\
\hline 19 & 11.8746 \\
\hline 26 & 4.6557 \\
\hline 30 & 7.1452 \\
\hline
\end{tabular}

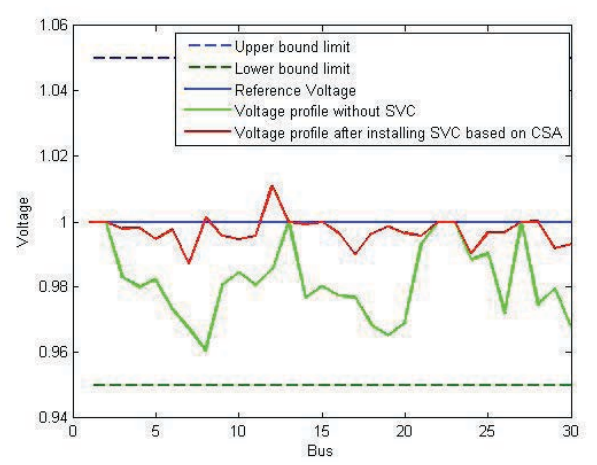

Figure 4. Voltage profiles of the best solution proposed by CSA in IEEE 30-bus case study 


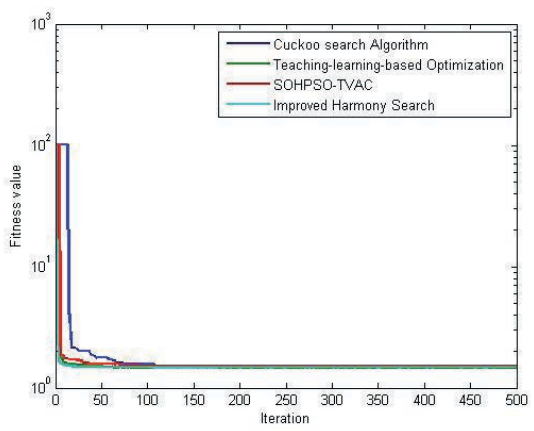

Figure 5. Comparison about convergences of proposed methods

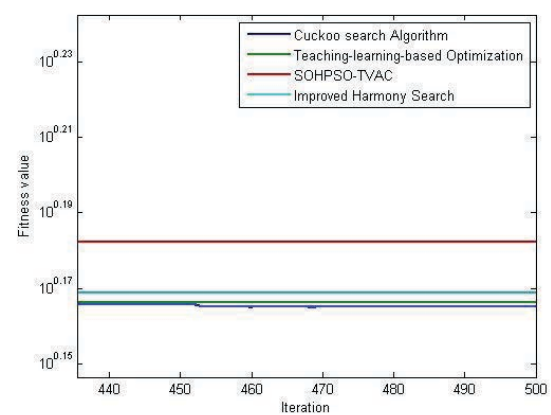

Figure 6. Zoomed image of convergences at the end of search process

According to numerical results in Table 3, Cuckoo search algorithm and TLBO give the same optimal solution and it is better than those given by SOHPSO-TVAC and IHS. However, in general, the Cuckoo search is better performance with lower average value and lower standard deviation.

Table 4 shows the best solutions proposed by Cuckoo search algorithm. Five selected buses are $8^{\text {th }}, 12^{\text {th }}, 19^{\text {th }}, 26^{\text {th }}$ and $30^{\text {th }}$ buses. After installing SVC, voltage magnitudes at these buses has been enhanced as Figure 4.

Figure 5 and 6 consider the convergence of these methods, where Figure 6 is a zoom image of Figure 5 at the end of calculating process. Cuckoo search algorithm starts slower than other methods. However, it reaches the best solution at the end of process. Its solution is slightlt better than the ones proposed by Teaching-learning-based optimization and Improved Harmony search.

\subsection{Case study 2: IEEE 57-bus system}

Table 5. Numerical results of compared methods for IEEE 57-bus system

\begin{tabular}{|c|c|c|c|c|}
\cline { 2 - 5 } \multicolumn{1}{c|}{} & CSA & TLBO & $\begin{array}{c}\text { SOHPSO } \\
\text { TVAC }\end{array}$ & IHS \\
\hline Best & 62.593 & 63.555 & 70.758 & 66.208 \\
\hline Mean & 68.119 & 70.279 & 91.184 & 101.794 \\
\hline Worst & 73.169 & 76.809 & 105.642 & 188.203 \\
\hline SD & 3.141 & 4.520 & 8.259 & 42.231 \\
\hline
\end{tabular}

Table 6. Optimal solution of CSA in IEEE 57-bus case study

\begin{tabular}{|c|c|}
\hline Selected bus & Reactive power [MVar] \\
\hline 20 & 7.6985 \\
\hline 31 & 5.0549 \\
\hline 35 & 22.1316 \\
\hline 42 & 6.5069 \\
\hline 47 & -49.9728 \\
\hline 51 & -31.7249 \\
\hline
\end{tabular}

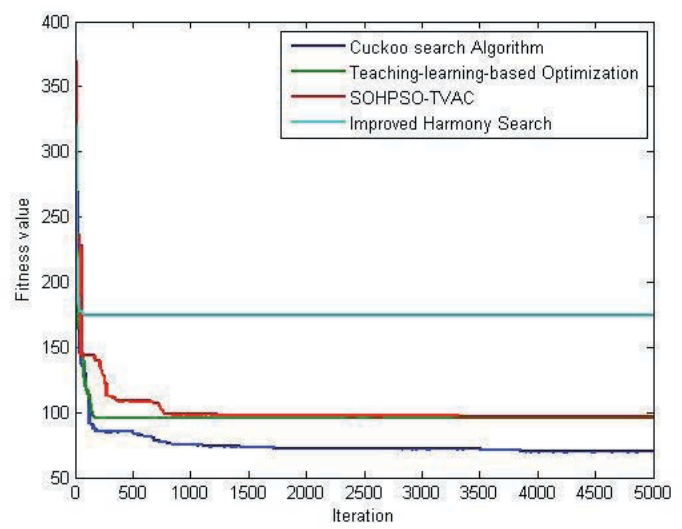

Figure 8. Comparison about convergences of CSA and TLBO

Table 5 shows the Monte Carlo numerical results. The Cuckoo search algorithm is clearly better than other compared search engines. The Cuckoo search algorithm does not only give better solutions, but its performance also is higher than the others. The best solution of CSA is given in Table 6 . Cuckoo search algorithm suggests to inject reactive power at the $20^{\text {th }}, 31^{\text {th }}, 35^{\text {th }}$ and $42^{\text {th }}$ buses and absorb reactive power at the $47^{\text {th }}$ and $51^{\text {th }}$ buses. After installing SVC, voltage magnitudes at the $31^{\text {th }}$ and $47^{\text {th }}$ buses have been enhanced as Figure 7 . 


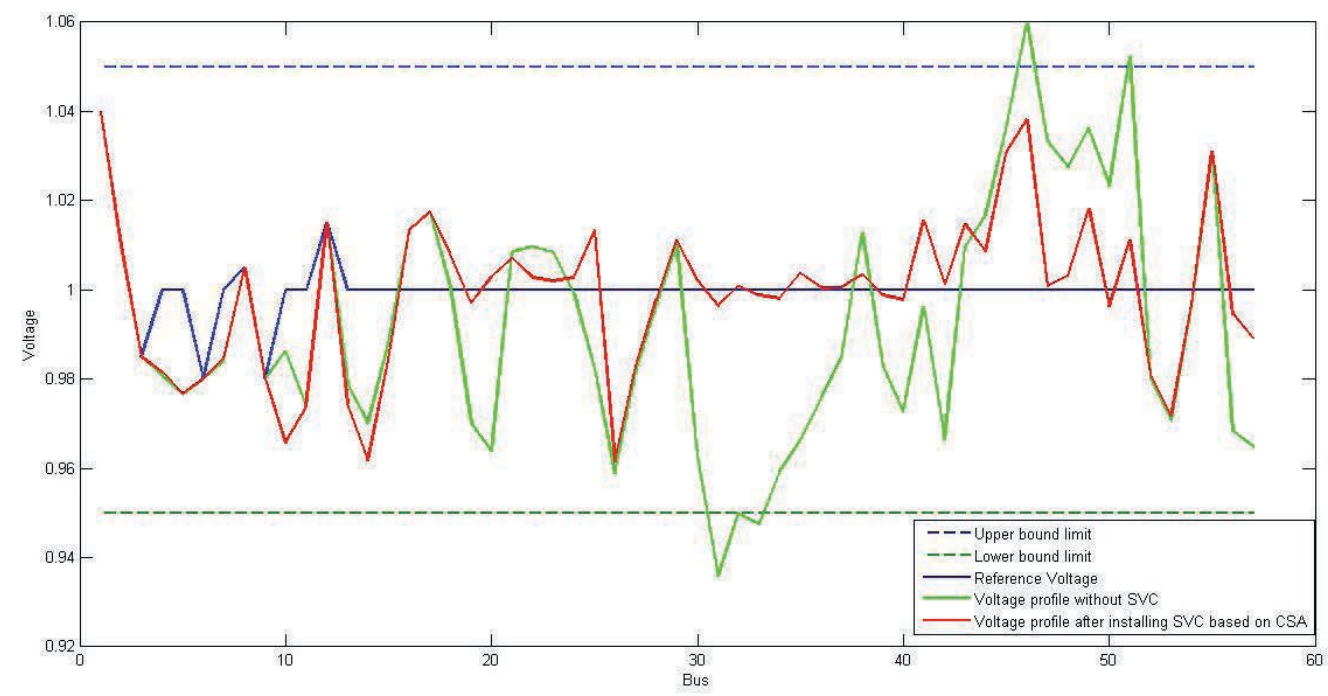

Figure 7. Voltage profiles of proposed methods in the IEEE 57-bus system

Table 7. Best results of compared methods for IEEE 118-bus system

\begin{tabular}{|c|c|c|c|c|c|c|c|c|}
\hline \multirow{2}{*}{$\begin{array}{c}\text { No. of } \\
\text { installed SVC }\end{array}$} & \multicolumn{2}{|c|}{ CSA } & \multicolumn{2}{c|}{ TLBO } & \multicolumn{2}{c|}{ SOHPSO-TVAC } & \multicolumn{2}{c|}{ IHS } \\
\cline { 2 - 9 } & $\begin{array}{c}\text { Selected } \\
\text { bus }\end{array}$ & $\begin{array}{c}\text { Reactive } \\
\text { power }\end{array}$ & $\begin{array}{c}\text { Selected } \\
\text { bus }\end{array}$ & $\begin{array}{c}\text { Reactive } \\
\text { power }\end{array}$ & $\begin{array}{c}\text { Selected } \\
\text { bus }\end{array}$ & $\begin{array}{c}\text { Reactive } \\
\text { power }\end{array}$ & $\begin{array}{c}\text { Selected } \\
\text { bus }\end{array}$ & $\begin{array}{c}\text { Reactive } \\
\text { power }\end{array}$ \\
\hline 1 & 2 & 50 & 2 & 50 & 21 & 41.0593 & 2 & 50 \\
2 & 13 & 50 & 13 & 50 & 37 & -2.5962 & 13 & 50 \\
3 & 20 & 50 & 14 & 32.4255 & 48 & 0.1190 & 14 & 50 \\
4 & 28 & 50 & 20 & 50 & 52 & 40.2274 & 20 & 39.5373 \\
5 & 53 & 50 & 28 & 50 & 53 & 9.8975 & 28 & 50 \\
6 & 58 & 50 & 39 & 50 & 57 & 19.4900 & 39 & -43.75 \\
7 & 95 & 50 & 52 & 50 & 58 & 37.3924 & 52 & 50 \\
8 & 106 & 50 & 109 & 50 & 75 & 27.9348 & 109 & 45.0496 \\
9 & 109 & 50 & 115 & 50 & 79 & -17.0275 & 115 & 41.1726 \\
10 & 115 & 50 & 118 & 50 & 84 & 11.8723 & 118 & 50 \\
\hline Best & \multicolumn{2}{|c|}{23.2405} & \multicolumn{2}{|c|}{23.9943} & \multicolumn{2}{|c|}{30.7140} & \multicolumn{2}{|c|}{31.6174} \\
\hline
\end{tabular}


According to Figure 8, it clearly shows that Cuckoo search algorithm is better than other methods to find the global optimum. All of TLBO, SOHPSO-TVAC and IHS are easily stuck in local optima.

\subsection{Case study 3: IEEE 118-bus system}

Once again, Cuckoo search algorithm gives better solution than the other methods. Detailed best solutions of compared methods are shown in Table 7. Both of the proposed method and the TLBO try to inject reactive power as much as possible but their proposed locations are different. However, the solution of Cuckoo search algorithm is slightly better than the one of TLBO, and clearly better than SOHPSO-TVAC and IHS.

\section{Conclusions}

The Cuckoo search algorithm is totally powerful and effective for determining location and size of SVC devices. Optimizing location and size of SVC devices is a complex problem. It combines continuous and discrete numbers with many equal and unequal constraints. It is easy to let the search engine to local optimums. However, according to three case studies, the Cuckoo search always gives the better solution with the higher performance. Comparing with Teaching-learningbased optimization, Cuckoo search algorithm may converge slower at the beginning, but it always give better solution at the end of search process. Comparing with SOHPSO-TVAC and IHS, Cuckoo search algorithm totally gives better solutions. On summary, the Cuckoo search algorithm is an effective optimization strategy to optimize location and size of SVC devices in a bulk power system. Furthermore, it is also favorable for the problem that combines continuous and discrete numbers.

\section{References}

[1] Del Valle, Y., Hernandez, J. C., Venayagamoorthy, G. K., \& Harley, R. G., Optimal STATCOM Sizing and Placement Using Particle Swarn Optimization. In Transmission \& Distribution Conference and Exposition: Latin America. TDC'06. IEEE/PES. IEEE, 2006, pp. 1-6.

[2] Chao-Ming, H., Yann-Chang H., Kun-Yuan H.
\& Hong-Tzer Y., A Harmony Search Algorithm for Optimal Power Flow Considering Flexible AC Transmission Systems, Intelligent Systems Applications to Power Systems, ISAP 2013. International Conference on, 2013, pp. 1-6

[3] Pisica, I., Bulac, C., Toma, L., \& Eremia, M., Optimal SVC placement in electric power systems using a genetic algorithms based method. In PowerTech, IEEE Bucharest. IEEE, 2009, pp. 1-6.

[4] Sirjani, R., Mohamed, A., \& Shareef, H., Optimal placement and sizing of Static Var Compensators in power systems using Improved Harmony Search Algorithm. Przeglad Elektrotechniczny, 87(7), 2011, 214-218.

[5] Sirjani, R., Mohamed, A., \& Shareef, H., Optimal allocation of shunt Var compensators in power systems using a novel global harmony search algorithm. International Journal of Electrical Power \& Energy Systems, 43(1), 2012, 562-572.

[6] Yang, X. S.,\& Deb, S., Cuckoo search via Lévy flights. In Nature \& Biologically Inspired Computing, 2009. NaBIC 2009. World Congress on (pp. 210-214). IEEE.

[7] Yang, X. S., \& Deb, S., Engineering optimization by cuckoo search. International Journal of Mathematical Modelling and Numerical Optimisation,1(4), 2010, 330-343.

[8] Civicioglu, P., \& Besdok, E., A conceptual comparison of the Cuckoo-search, particle swarm optimization, differential evolution and artificial bee colony algorithms. Artificial Intelligence Review, 39(4), 2013, 315-346.

[9] Moravej, Z., \& Akhlaghi, A., A novel approach based on cuckoo search for DG allocation in distribution network. International Journal of Electrical Power \& Energy Systems, 44(1), 2013, 672-679.

[10] Ahmed, J., \& Salam, Z., A Maximum Power Point Tracking (MPPT) for PV system using Cuckoo Search with partial shading capability. Applied Energy, 119, 2014, 118-130.

[11] Vo, D. N., Schegner, P., \& Ongsakul, W., Cuckoo search algorithm for non-convex economic dispatch. Generation, Transmission \& Distribution, IET,7(6), 2013.

[12] Cai, L. J., Erlich, I., \& Stamtsis, G., Optimal choice and allocation of FACTS devices in deregulated electricity market using genetic algorithms. In Power Systems Conference and Exposition, 2004. IEEE PES(pp. 201-207). IEEE.

[13] Mantegna, R. N., Fast, accurate algorithm for numerical simulation of Levy stable stochastic processes. Physical Review E, 49(5), 1994, 4677. 
[14] Zimmerman, R. D., Murillo-Snchez, C. E., \& Thomas, R. J., MATPOWER: Steady-state operations, planning, and analysis tools for power systems research and education. Power Systems, IEEE Transactions on,26(1), 2011, 12-19.

[15] Rao, R. V., Savsani, V. J., \& Vakharia, D. P., Teachinglearning-based optimization: an optimization method for continuous non-linear large scale problems. Information Sciences, 183(1), 2012,1-15.

[16] Rao, R., \& Patel, V. (2012). An elitist teachinglearning-based optimization algorithm for solving complex constrained optimization problems. International Journal of Industrial Engineering Computations, 3(4), 535-560.

[17] Ratnaweera, A., Halgamuge, S. K., \& Watson, H. C. (2004). Self-organizing hierarchical particle swarm optimizer with time-varying acceleration coefficients.Evolutionary Computation, IEEE Transactions on, 8(3), 240-255.

[18] Mahdavi, M., Fesanghary, M., \& Damangir, E. (2007). An improved harmony search algorithm for solving optimization problems. Applied mathematics and computation, 188(2), 1567-1579

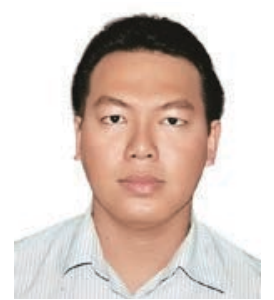

Khai Phuc Nguyen received his B.Eng. and M.Eng. degrees in electrical engineering from Ho Chi Minh City University of Technology, Vietnam, in 2010 and 2012, respectively. $\mathrm{He}$ is a Ph.D. student at Shibaura Institute of Technology, Tokyo, Japan. His research interests Artificial Intelligence (AI) in power system optimization, power system operation and control, power system analysis and automation in power system.

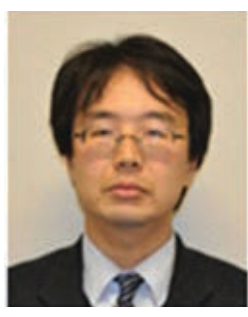

Goro Fujita received the B.Eng., M.Eng. and Ph.D. degrees in electrical engineering from Hosei University, Tokyo, Japan in 1992, 1994 and 1997, respectively. He is a Professor at Shibaura Institute of Technology, Tokyo, Japan. His interest is in power system control including dispersed power systems. He is a Professional Engineer in Japan and First Class Licensed Engineer of Electric Facility.

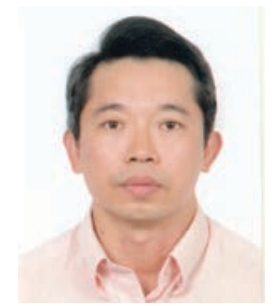

Vo Ngoc Dieu received his B.Eng. and M.Eng. degrees in electrical engineering from Ho Chi Minh City University of Technology, Ho Chi Minh city, Vietnam, in 1995 and 2000, respectively and his D.Eng. Degree in energy from Asian Institute of Technology (AIT), Pathumthani, Thailand in 2007. He is Research Associate at Energy Field of Study, AIT and Head of Department of Power Systems, Faculty of Electrical and Electronic Engineering, Ho Chi Minh City University of Technology, Ho Chi Minh city, Vietnam. His research interests are applications of AI in power system optimization, power system operation and control, power system analysis, and power systems under deregulation and restructuring. 